\title{
Development and implementation of a stroke rehabilitation integrated care pathway in an Italian no profit institution: an observational study
}

\author{
Francesca CECCHI 1, Manuela DIVERIO 1, Chiara ARIENTI 2, Elena CORBELLA 2, \\ Federico MARRAZZO 1, Gabriele SPERANZA 1, Elisabetta DEL ZOTTO 2, Giuliana POGGIANTI 1, \\ Francesco GIGLIOTTI 1, Paola POLCARO 1, Margherita ZINGONI 1, Desiderio ANTONIOLI 1, \\ Lucia AVILA 1, Manuele BARILLI 1, Emanuela ROMANO 1, Lucilla LANDUCCI PELLEGRINI 1, \\ Massimo GAMBINI 1, Sonia VERDESCA ${ }^{1}$, Federica BERTOLUCCI ${ }^{1}$, Irene MOSCA ${ }^{1}$, \\ Paola GEMIGNANI 1, Anita PAPERINI ${ }^{2}$ *, Chiara CASTAGNOLI 1, Ines HOCHLEITNER 1, \\ Maria L. LUISI 1, Giulia LUCIDI 1, Bahia HAKIKI 1, Maria A. GABRIELLI 2, \\ Morena FRUZZETTI 1, Annalisa BRUZZI 1, Enrico BACCI BONOTTI 1, Silvia PANCANI 1, \\ Silvia GALERI ${ }^{2}$, Claudio MACCHI ${ }^{1}$, Irene APRILE ${ }^{1}$
}

${ }^{1}$ IRCCS Fondazione Don Carlo Gnocchi, Florence, Italy; ${ }^{2}$ IRCCS Fondazione Don Carlo Gnocchi, Milan, Italy

*Corresponding author: Anita Paperini, IRCCS Fondazione Don Carlo Gnocchi, Via di Scandicci 269, 50143 Florence, Italy.

E-mail: apaperini@dongnocchi.it

\section{A B S T R A C T}

BACKGROUND: To standardize assessment and coordinate processes in stroke rehabilitation, an integrated care pathway (ICP) was developed in an Italian Rehabilitation and Research Institution by a knowledge-translation interdisciplinary process, from evidence-based guidelines to rehabilitation practice. The ICP was implemented in two pilot Tuscan rehabilitation Centers.

AIM: The purpose of this study was to describe ICP development and assess the ICP effects on postacute stroke inpatient rehabilitation outcomes. DESIGN: Prospective observational study, before and after comparison.

SETTING: Two Tuscan inpatient rehabilitation centers.

POPULATION: Patients accessing either centers for intensive rehabilitation after acute stroke.

METHODS: Two cohorts were prospectively recruited before (2015-2017) and after (2018) implementation of the pathway. The primary outcome was change in activities of daily living disability, assessed by the modified Barthel Index (mBI) from admission to discharge. Secondary outcomes included length of stay (LOS), adverse outcomes, and changes in communication ability, trunk control, pain, ambulation, bladder catheter $(\mathrm{Y} / \mathrm{N})$, bedsores $(\mathrm{Y} / \mathrm{N})$.

RESULTS: In 2015-2017, 443 postacute stroke patients (mean age $77 \pm 11$ years, $47 \%$ women), while in 2018,84 patients (mean age $76 \pm 13$ years, $61 \%$ women) were admitted to the two facilities. Comparing the $2018 v s$. the 2015-17 cohort, the mean mBI increase was not substantially different ( 26 vs. 24 points), nor were LOS ( $37 \pm 18$ vs. 36 \pm 16 days), adverse outcomes, discharge destination, and improvement of ambulation, pain, and communication ( $\mathrm{P}>0.05)$. Instead, a significantly higher improvement of trunk control (trunk control test: $69.6 \pm 33.2$ vs. 79.0 31.3 , $\mathrm{P}=0.019)$, and a higher percentage of bedsore resolution $(13 \%$ vs. $5 \%, \mathrm{P}=0.033)$, and bladder catheter removal $(37 \%$ vs. $17 \% \mathrm{P}<0.001)$ were observed in 2018 vs. 2015-2017.

CONCLUSIONS: Compared to prior practice, ICP was associated to improvement of trunk control recovery, bladder catheter removal, and bedsores resolution. Further ICP implementation on a larger scale is needed to verify improvements of stroke inpatient rehabilitation outcomes. CLINICAL REHABILITATION IMPACT: An evidence-based stroke rehabilitation ICP was interdisciplinary developed and implemented in two rehabilitation centers of a multicenter Italian health group. ICP implementation as to inpatient intensive postacute stroke rehabilitation was associated to improved trunk control recovery, bladder catheter removal, and bedsore resolution. Further ICP implementation will allow multicenter studies and quality benchmarking.

(Cite this article as: Cecchi F, Diverio M, Arienti C, Corbella E, Marrazzo F, Speranza G, et al. Development and implementation of a stroke rehabilitation integrated care pathway in an Italian no profit institution: an observational study. Eur J Phys Rehabil Med 2020;56:713-24. DOI: 10.23736/ S1973-9087.20.06195-X)

KEY WORDS: Delivery of health care, integrated; Stroke rehabilitation; Evidence-based practice; Quality improvement. 


\section{COPYRIGHT $^{\circledR} 2020$ EDIZIONI MINERVA MEDICA}

$T^{1}$ he past decade has seen great progress in the treatment of cerebrovascular diseases in the acute phase, but stroke remains a catastrophic event of relevant public health consequence, causing 5 million deaths each year and a much larger number of persons surviving with chronic disability worldwide. ${ }^{1}$ More than two thirds of stroke survivors experience post stroke disability, ${ }^{2}$ and the recovery of participation is even more challenging, with $>30 \%$ of them reporting persistent restrictions by four years after stroke onset, ${ }^{3}$ even when attending postacute stroke rehabilitation. ${ }^{4}$ As recently stated by the World Health Organization "mortality and morbidity from stroke could be significantly reduced through organized stroke care, including the implementation of evidence-based clinical practice guidelines and adoption of a continuous quality improvement philosophy and programs." 5 However, in a framework of continuous quality improvement, intended to better meet patient's needs by focusing on work processes and systems, ${ }^{6}$ rehabilitation strategies should rely on evidence-based standardized assessment protocols and care processes, allowing benchmarking of treatments on large populations of stroke patients. ${ }^{7,8}$ Yet, systems of care, approaches to stroke rehabilitation delivery, and outcome assessment, as well as resources for stroke care and rehabilitation, are still extremely variable across geographic regions worldwide. ${ }^{9}$

In Italy, stroke rehabilitation recommendations make general reference to national and international guidelines, ${ }^{10}$ but their operational definition to protocols and pathways is very heterogeneous and significantly affected by the different standards applied at regional and even local level, creating a different flow of patients within the healthcare system at different local levels, with high risk for inequalities and for suboptimal care.11 Further, although in 2005 the Italian Society of Physical and Rehabilitation Medicine proposed a minimum assessment protocol for post-stroke patients; 12 there is no National Health System requirement as to measures of stroke rehabilitation outcomes. Thus, to date, there is no standard quality assessment for the benchmarking of different rehabilitation facilities and approaches.

Quality improvement (QI) in stroke rehabilitation requires a standardization of outcome assessment and of processes, to provide opportunity for local, regional and global benchmarking of stroke care delivery. ${ }^{13}$ A growing amount of evidence suggests that a tool to QI in complex care processes such as rehabilitation may be represented by integrated care pathways (ICPs) ${ }^{14}$ proposed in the USA since the eighties to improve the flow of patients within the healthcare system. ICPs are a complex intervention for the mutual decision making and organization of care processes for a well-defined group of patients during a welldefined period. ${ }^{15}$ They are designed and implemented to map the patient journey to enable the right people to do the right things, in the right order, at the right time, in the right place, with the right outcome. ${ }^{16}$ ICPs aim at identifying all necessary care elements and standardize care trajectory processes, though variances allow for professional judgment and personalized care. ${ }^{16}$ They can provide positive effects in improving quality and expediency of clinical processes. ${ }^{14}$ As to stroke, ICPs are reported to increase patient satisfaction and promote consistent, collaborative multidisciplinary care, ${ }^{17}$ while it is yet not clear whether they lead to better clinical, functional, and economic outcomes. ${ }^{14}$

In the framework of continuous QI, ${ }^{6}$ the National Direction of the Don Carlo Gnocchi Foundation (FdG), an Italian no-profit rehabilitation and research institution, active in Northern, Central, and Southern Italy, in 2016, appointed a Committee of Rehabilitation Professionals. The aim of the committee was to evaluate, compare and improve complex care processes and outcomes, and develop consensus over evidence-based ICPs of complex disabilities, in a continuous process of knowledge translation in physical and rehabilitation medicine from evidence-based guidelines to rehabilitation practice.

The aim of this study was to describe the development and implementation of an evidence-based interdisciplinary developed ICP for postacute stroke inpatient rehabilitation, inside two FdG Centers, and to assess the ICP effects on postacute stroke inpatient rehabilitation outcomes.

\section{Materials and methods}

\section{ICP development}

All participants were appointed for their expertise in the field from five different FdG Rehabilitation Centers located in Lombardy, Tuscany, Liguria, Basilicata, Campania. The original focus group involved in designing the ICP included: 6 medical doctors (MDs) (five physiatrists and one neurologist expert in rehabilitation). Interdisciplinary ICP development required early (June 2016) inclusion of other rehabilitation professionals: four physiotherapists (PTs), one nurse, one occupational therapist, one speech therapist, two psychologists. The pathway was developed as a process of knowledge translation of evidence-based recommendations to rehabilitation practice. First, an extensive review of existing protocols and regional ICPs, 


\section{COPYRIGHT $^{\circledR} 2020$ EDIZIONI MINERVA MEDICA}

and multiple consultations with experts and care providers of all the involved centers were carried out, to analyze current practice, barriers, and incentives for change. ${ }^{18}$ Then, a shared reference framework for the ICP definition was identified. In agreement with international and national recommendations, the reference frame for defining domains of body functions, Activity, and participation was the international classification of functioning, disability and health. ${ }^{19}$ Operating in Italy, the Italian Ministry of Health requirements and recommendations were also taken into account. ${ }^{9}, 10$ Among relevant international and national guidelines on stroke rehabilitation, ${ }^{20,21}$ the chosen reference guidelines were the American Heart Association/American Stroke Association (AHA/ASA) guidelines for stroke rehabilitation, published in $2016,{ }^{4}$ as they were the most recently updated and scored as "high quality," according to the Appraisal of Guidelines for Research and Evaluation Instrument, revised (AGREE II). ${ }^{22}$ Outcome measures were selected based on AHA/ASA guidelines, on the indicators of process and outcome and rehabilitation 2 -IPER 2 model, ${ }^{23}$ and on specific review of the literature on the gold standard measures in stroke rehabilitation. $^{4,24}$ For the analysis of the needs and the drafting of the individual rehabilitation project (IRP), to identify the objectives and the program with the activation of targeted therapeutic actions, reference was made to the areas of functioning that may be targets for the IRP interventions, as defined by Basaglia, ${ }^{25}$ systematically exploring: 1) clinical stability; 2) basic vital functions; 3 ) sensorimotor function; 4) mobility and transfers; 5) communication skills-relational; 6) cognitive-behavioral; 7) autonomy and self-care; 8) re-adaptation and social reintegration; and 9) emotional-affective, in line with the ICF model. ${ }^{19}$

Each ICP statement was collectively reviewed and approved first by all group members and then by the FdG national medical direction in May 2017.

Finally, in January 2018, the ICP was collectively revised as an assignment to all FdG health professionals participants to the Italian Group for evidence based medicine course: "From guidelines to care pathways," dedicated to the conceptual and methodological aspects of the correct drafting of an ICP;26 the contents and references were unaltered, but specific reference to ICP statements was also provided. The final revised stroke rehabilitation ICP was then officially approved by the medical direction in January 2018. This final approved version of the ICP, adopted for this pilot study, is summarized in Figure 1 and reported in detail in Supplementary Digital Material 1: Supplementary Text 1.

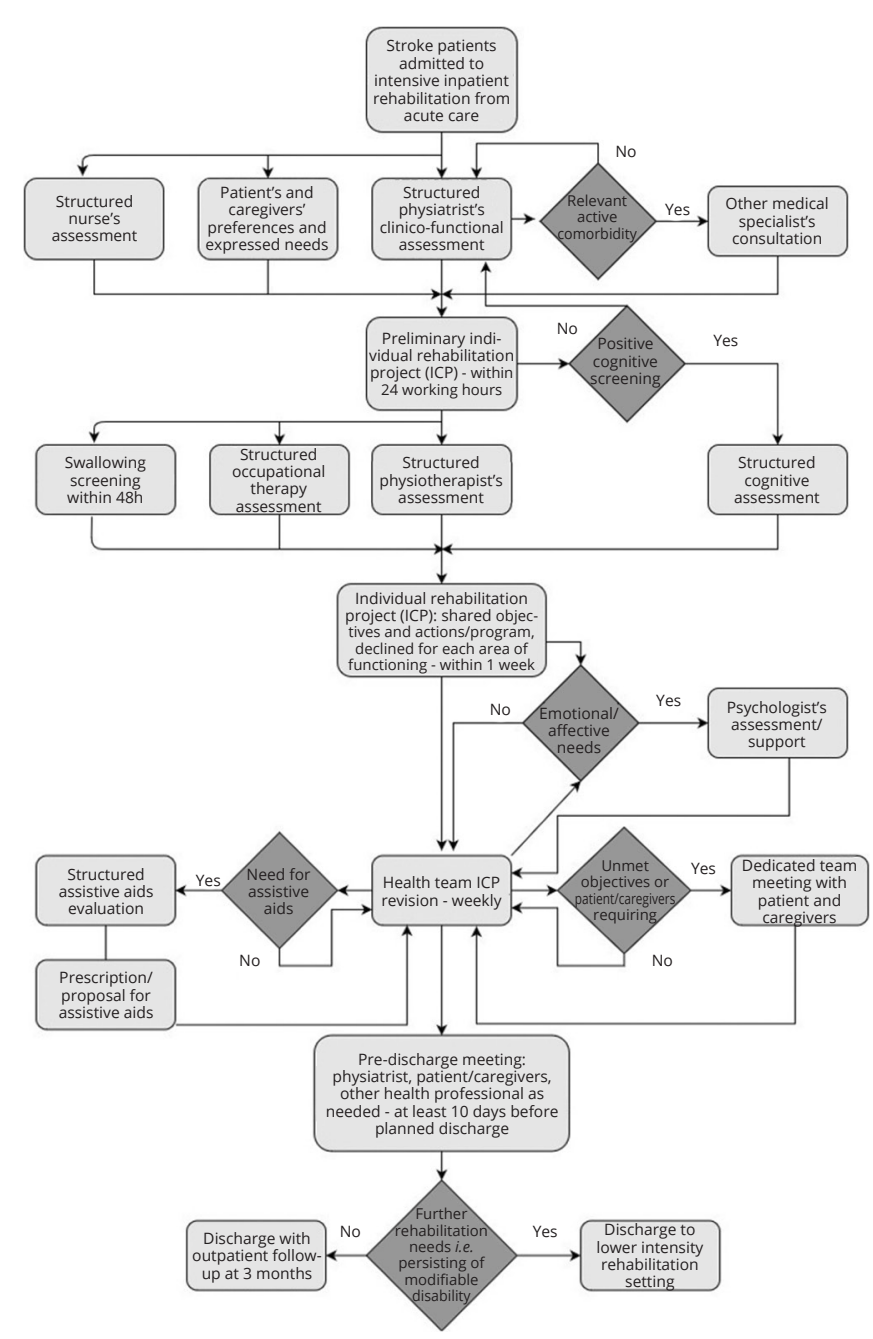

Figure 1.- The Fondazione Don Carlo Gnocchi inpatient stroke rehabilitation integrated care pathway (ICP).

\section{Study design}

An observational study was carried on verifying the feasibility and outcomes of the Stroke Rehabilitation ICP to carry on an observational study, upon approval of the direction and medical directions of the two centers.

\section{Setting}

The neurologic/orthopedic rehabilitation centers of Marina di Massa and Fivizzano, Don Gnocchi Foundation, are located in the province of Massa Carrara, Tuscany, Italy, and are the only accredited provider of inpatient rehabilitation for all the Massa-Carrara area (New Apuan Hospital and Fivizzano and Pontremoli Hospitals): Massa has 78 intensive rehabilitation beds, while Fivizzano has 32. 


\section{COPYRIGHT $^{\circledR} 2020$ EDIZIONI MINERVA MEDICA}

$\mathrm{CECCHI}$

IMPLEMENTATION OF A STROKE REHABILITATION ICP

\section{Patients}

From January 1, 2015 to June 30, 2018, all patients with stroke accessing the Don Gnocchi Rehabilitation facility of Marina di Massa and Fivizzano for intensive inpatient rehabilitation, by authorization of the local public health authority specialists in physical medicine and rehabilitation, were consecutively assessed.

In the framework of continuous quality assessment and QI, all patients on admittance were routinely informed and gave written consent to anonymous treatment of their personal and clinical data for clinical and research purposes. Patients' next of kin were informed and asked to sign the consent when the patient was not able to understand or to sign. Since the ICP was only an update of the previous protocols and procedures, in line with most recent evidence-based rehabilitation guidelines, no ethical issue was involved, and no Ethical Committee authorization was required.

\section{Procedure}

The steps for implementing the protocol were designed according to the Medical Research Council recommendations to promote the implementation of complex interventions in heath organizations ${ }^{27,} 28$ addressing the following key factors: organizational commitment, including document format, additional support and safety management, pathway content using evidence-based guidelines, multidisciplinary involvement in pathway maintenance. Many rehabilitation health professionals in the two facilities had been already formally involved in the ICP development, and the whole staff was given opportunity in formal and informal meetings to learn about the ICP and to produce comments and suggestions before its final approval and implementation.

\section{Intervention}

Inpatient Rehabilitation before and after ICP implementation

According to the Italian Ministry of Health and Tuscany regional health system requirements, stroke rehabilitation was already based on the Individual Rehabilitation Project (IRP), defined by an interdisciplinary team coordinated by the physiatrist, and revised, systematically, at weekly team meetings and as needed a delivering at least 3 hours of rehabilitation per day; the PT and nurse were always involved, while other health professionals were activated by the physiatrist according to the team identification of the patient's needs. Patients' satisfaction was collected by an anonymous written questionnaire.
Since January 2015, the structured medical and nurse assessment on admission and discharge already included for all rehabilitation patients: comorbidity, measured by the cumulative illness rating scale (CIRS), ${ }^{29}$ cognitive functioning, measured by the mini mental state examination (MMSE), ${ }^{30}$ trunk control, measured by the trunk control test (TCT), ${ }^{31}$ ambulation, measured by the standardized audit of hip fracture in Europe (SAHFE), ${ }^{32}$ communication ability, measured by the Scale of Communicative Disability $(\mathrm{SDC}),{ }^{33}$ pressure sores $(\mathrm{Y} / \mathrm{N})$ presence and stage (Agency for Health Care Policy and Research, AHCPR), ${ }^{34}$ risk of pressure ulcers, measured by the Braden Score, ${ }^{35}$ and pain, assessed by the numeric rating scale (NRS)PAIN, 36 ranging from 0 (no pain) to 10 (the worst possible pain). Functional evaluation, as required by local health authority, was measured by the modified Barthel Index $(\mathrm{mBI}){ }^{37,38}$ For stroke patients we also assessed motricity, measured by the Motricity Index (MI). ${ }^{39}$

Since January 2018, according the ICP, the following innovations were implemented:

- Assessment:

- to provide a continuous functional assessment with the acute care setting, we adopted the modified Rankin scale (mRankin), ${ }^{40}$ a measure of disability in basic and instrumental activities of daily living, not only as an anamnestic report, but also as an admission and discharge assessment measure;

- the gold standard measure of post-stroke neurologic impairment, also often applied in acute care settings, the National Institutes of Health Stroke Scale (NIH-SS), ${ }^{41,} 42$ was introduced in the admission and discharge medical assessment;

- a classification and of cerebrovascular territory involved in ischemic stroke, through the Oxfordshire Community Stroke Project (OCSP) ${ }^{43}$ were introduced at admission;

- since NIH-SS included some cognitive items and that communication was also assessed by SDC, MMSE was no longer required;

- the protocol also required the physiotherapists to administer the Motricity Index (MI) and the short physical performance battery (SPPB), ${ }^{44}$ as an index of lower limbs performance to integrate functional assessment with a measure of balance, raising from/sitting on a chair, and $4 \mathrm{~m}$ walking ability.

- standardization of minimum requirements for medical assessment and exams on admission;

- systematic report in clinical records of the IRP development according to the ICF model through a systematic 


\section{COPYRIGHT $^{\circledR} 2020$ EDIZIONI MINERVA MEDICA}

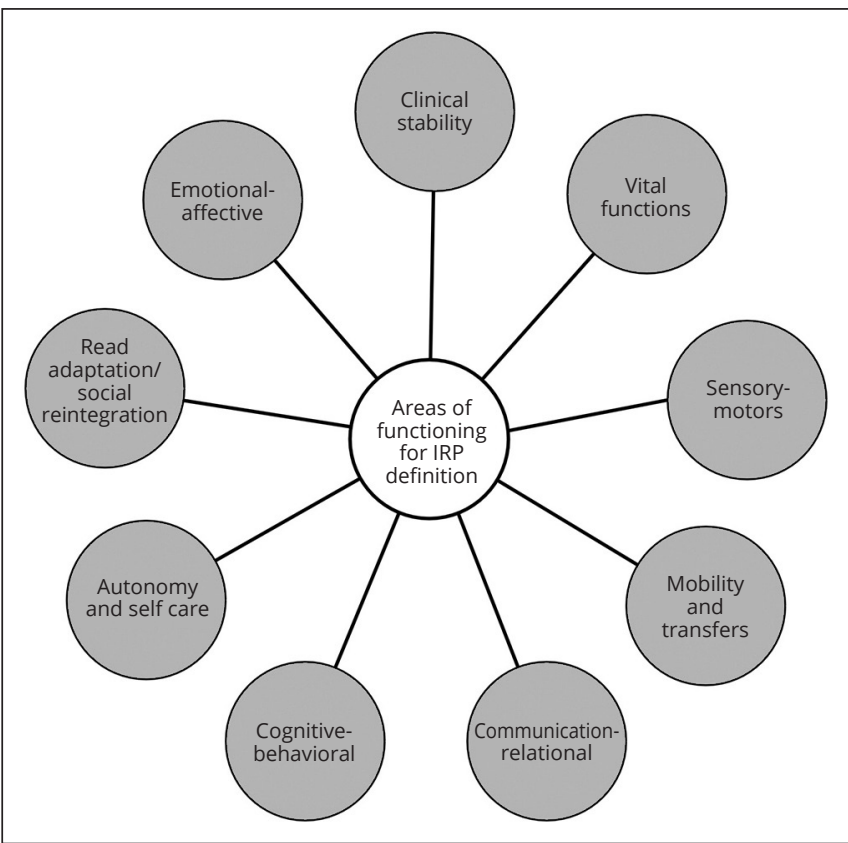

Figure 2.-The areas of functioning for individual rehabilitation project (IRP) definition.

description of the patient's needs and of the relative objectives and therapeutic actions/program, according to the areas of functioning/targets for IRP intervention, as described by Basaglia 25 (Figure2);

- systematic monitoring and interdisciplinary rediscussion of patient-centered objectives at weekly team meetings, coordinated by the physiatrist directing both facilities, or by another team physiatrist, according to the same framework;

- team meetings involving always a nurse and a healthcare assistant;

- dedicated (focus) team meetings with patients and/or caregivers when any unmet objective, including difficulty in communication, collaboration, or any problem related to prospected discharge would arise during the stay;

- strict timing of preliminary IRP definition (physiatrist, nurse, and physiotherapist coordinator) within the second working day from admittance;

- systematic screening for dysphagia and for referral to dysphagia and/or speech therapy (water swallow test within 2 working days from admittance, NIH-SS; SDC);

- systematic cognitive screening at admission (NIHSS, SDC, clinical assessment);

- systematic referral to occupational therapy assessment;

- Explicit assignment of responsibility to different health professionals according to different areas and functions involved.

The intervention is summarized by the TIDIeR checklist. ${ }^{45}$ For more details on the ICP, see Supplementary Text 1.

\section{Protocol promotion and implementation}

The steps for promoting the protocol were designed according to promote change in a health organization, thus we focused on public knowledge, direction and health staff commitment, identification of change leader/change leading team, team communication and education, and performance management, by planning systematic monitoring, feedback and coaching. ${ }^{27,28}$ Planned steps included:

- analysis of outcomes of stroke in-patients in the 3 years prior to implementation;

- identification of the change leader (C.F., coauthor of the ICP, physiatrist directing both facilities) and change leading team (G.M.A., coauthor of the ICP and coordinator of all non-medical health staff, plus all the $10 \mathrm{MDs}$ working in either facility, as one of them always conducted ward team meetings in the absence of the change leader);

- presentation to and request of preliminary approval by the Direction and Medical Direction of Massa and Fivizzano of the Stroke ICP;

- presentation and discussion of the stroke protocol to both facilities' health professionals and training on the assessment measures' administration;

- review of health records and official stroke rehabilitation protocols (declared to the local health authority);

- development of a database for selected assessment measures and rehabilitation outcomes;

- implementation;

- systematic monitoring, feedback and coaching by the change leader or by a member of the change team at weekly team meetings: during weekly team meetings, all clinical records would be checked one by one and each professional's difficulty with any tool's administration discussed; to discuss eventual difficulties, dedicated audits could be activated by each member of the change team or of the health staff;

- comparing outcomes with 2015-2017 outcomes after 6 months from implementation;

- ICP critical appraisal, shared with health professionals and possible ICP revision after 6 months from implementation.

\section{Outcome measures}

For ICP feasibility and staff acceptability, chosen indicators included: 1) clinical records compilation (weekly 


\section{COPYRIGHT $^{\circledR} 2020$ EDIZIONI MINERVA MEDICA}

checked one by one by the change team leader or by a member of the change) and specifically, NIH-SS compilation on $\geq 60 \%$ stroke patients in the 6 month time of observation; 2) number of audits required by the chance team or the health staff to discuss difficulties in ICP implementation in the 6 month time of observation; 3 ) timely adoption of the organizational changes required; and 4) evidence of adherence to interdisciplinary care provided by weekly team meetings.

As to patient-oriented outcomes, for all patients, functional and clinical measures were recorded at admission and at discharge, by the above mentioned validated and standardized tools, shared with the local health authority. Patients' satisfaction was anonymously reported in a dedicated questionnaire.

Admission data included personal data, comorbidity, assessed by the CIRS and cognitive level (MMSE), and prestroke functional level, assessed by the premorbid modified Rankin Scale. For patients enrolled since January 2018, type of ischemic stroke (OCSP), laterality, thrombolysis, stroke related impairment (NIH-SS) were also recorded, while MMSE was no more required.

The primary rehabilitation outcome was change in activities of daily living disability, as assessed by the $\mathrm{mBI}$, ranging from 100 (no disability) to 0 . Other selected rehabilitation outcomes included length of stay (LOS), percentage of adverse outcomes (deaths or discharge to acute care hospital), and functional and clinical indicators, recorded on admission and at discharge: presence of the urinary catheter $(\mathrm{Y} / \mathrm{N})$, presence of bedsores $(\mathrm{Y} / \mathrm{N})$, disability in communication (SDC), trunk control (TCT), pain (NRS), ambulation (SAHFE), and, only for patients enrolled since January 2018, improvement of neurologic impairment (NIH-SS).

\section{Statistical analysis}

Data were analyzed using IBM SPSS Statistics 24.0 for Windows. For each considered tome interval (2015-2017 and 2018), Shapiro-Wilk test was used to verify if parameters were normally distributed (significant deviation from normality was assumed in presence of a $\mathrm{P}$ value $<0.05$ ). According to the test results, parameters characterized by a normal distribution were represented by mean and standard deviation, parameters with a non-normal distribution by median and interquartile range, dichotomous parameters by percentage. Clinical and functional parameters measured at admission were compared to those measured at discharge by using a paired $t$-test for continuous, normally distributed variables, a Wilcoxon signed rank test for ordinal and not normally distributed variables and a McNemar test for dichotomous variables. Differences in parameters between the two-time intervals under observation (2015-2017 vs. 2018) were investigated using an independent $t$-test for continuous variables and a $\chi^{2}$ test for dichotomous variables. However, to ensure that the unequal sample sizes between the two time periods would not affect statistical significance, a bias-corrected bootstrapping procedure based on 2000 bootstrap samples was applied to establish the achieved significance level. ${ }^{46} \mathrm{~A} P$ value $<0.05$ was chosen for statistical significance. Only trivial differences were observed between P values derived from the $t$ test and bootstrap analyses. In particular, no conclusion of significance was dependent on the choice of statistical test.

\section{Results}

\section{Protocol promotion and implementation}

The steps to promote and implement the study protocol were realized as follows:

- presentation of the ICP in the training course: assessment as a basis for rehabilitation treatment addressed to all health staff (two editions, second semester 2017);

- team building meetings including the change leader ad all team medical staff chosen as the change team (weekly, from October to February 2017);

- ICP discussion in 6 dedicated meetings including all medical specialists, PT, occupational therapists, speech therapists, and psychologists (second semester 2017);

- approval of the Health Direction of the clinical record revision to include new clinical and outcome measures and identifications of needs and objectives according to the 9 domains of functioning explored 22,23 (December 2017);

- database revision to include new assessment measures (January 2018);

- online certification for NIHSS administration for all MD specialists (January 2018); ${ }^{42}$

- ICP implementation: organizational changes start, use of revised clinical records (January 2018).

Feasibility and acceptability

Changes in the organization were introduced right at ICP implementation (January 2018). No formal cost assessment was performed, but we calculated that training course was included into the 2017 continuous education program, and the clinical records were modified before their reprint was due, so that no additional expense was required. The only live cost to the organization was related to all MD 


\section{COPYRIGHT $^{\circledR} 2020$ EDIZIONI MINERVA MEDICA}

spending 4 hours to complete NIH-SS online certification $(4 \times 10=40$ hours $\times$ Euro $36=$ Euro 1440$)$. To allow systematic dysphagia assessment, the speech therapists schedule was rearranged to allow 1 speech therapist always supervising lunchtime, thus assessing and reassessing one to 3 patients in $45^{\prime}$. Systematic referral to occupational therapy required to shift a part time PT already formed in occupational therapy to support the occupational therapy team; the overall time devoted to rehabilitation stayed the same and no additional expense was due to these organizational changes. Measures required in the clinical records were routinely recorded since January 2018. Although we did not formally measure the staff perceptions as to the implemented ICP, feedback on ICP implementation was constantly provided at weekly team meetings and at weekly change team meetings. The ICP was well accepted by the health staff and no dedicated audit was required by either the change team of the staff; only 1 audit was activated by the change leader to rediscuss with all MDs the NIH-SS administration after 2 months from ICP implementation. At the finale critical revision, all MDs expressed appreciation of the NIH-SS, as providing a structured, repeatable and informative neurological assessment of stroke patients. Psychologists, nurses and health assistants reported to appreciate the interdisciplinary approach proposed. However, although PTs were involved in the choice of assessment measures and agreed to the ICP implementation, because of organizational problems, unrelated to the ICP implementation (i.e. lack of authorization from the local direction to change PT records before changes were agreed throughout all Tuscan and Ligurian centers), the new PT assessment was not included in the PT records. As to patients' satisfaction, the anonymous questionnaires reported positive feedback, but, as in 2015-2017. It was completed only by $15 \%$ cases; no significant changes as to voluntary discharge were either observed (see below), but we lack a direct, reliable measure of patients' satisfaction.

\section{Adherence to implementation}

The medical head of the facilities systematically checked clinical records for assessment completion at weekly team meetings; when some assessments were missing, the health professionals were invited to complete them within the next week. After the first 2 months, since the compilation of the NIH-SS was not systematic, one audit involving all MD was held, and the administering procedure was re-discussed in detail. Ultimately, the NIH-SS was administered to $64 \%$ patients in the 6-month observation time, with all records complete in the last two months. While in
Fivizzano the healthcare assistant regularly participated to team meetings, in Massa this was sometimes unattended. The PT record was not reprinted because of organizational problems, unrelated to ICP implementation, thus the PT scales were not administered (MI, SPPB)

Comparison of case mix and rehabilitation outcome before and after ICP implementation

Between 2015 and 2017, 443 stroke patients were admitted to the rehabilitation center; from January 15 to June 30, 2018, 84 stroke patients were admitted. Among 48 ischemic stroke patients, 6 were classified by the OCSP as TACS: total anterior circulation; 14 as PACS: partial anterior circulation; 11 as POCS: posterior circulation; 8as LACS: lacunar syndromes, and 10 had missing values. Patients were admitted 13.5 days (median value) after stroke onset; this information was not available for the reference period 2015-2017. Patients admitted for stroke rehabilitation in the three-years period 2015-2017 and in the first semester of 2018. Clinical and functional characteristics registered at admission and discharge are shown in Table I. Patients' admission characteristics were similar except for a higher prevalence of women in the 2018 sample.

Comparing admission to discharge measures, the mean mBI significantly increased from admission to discharge by 26 points in 2018 , and by 24 points in 2015-17. The average LOS in 2018 was $37 \pm 18$ days, in line with that observed in 2015-17 (36 \pm 16 days). SAHFE, pain NRS and SDC scores were also significantly improved from admission to discharge both in 2018 and in 2015-17, but with similar observed differences. On the other hand, the TCT score significantly increased from admission to discharge by 33 points vs. 21 points in $2018 v s$. 2015-17. In 2018, patients requiring a bladder catheter on admission were $52 \%$, and $15 \%$ on discharge, while in $2015-17$ they were $34 \%$ on admission, and $17 \%$ on discharge. Bedsores on discharge were significantly less than what observed on admission, both in 2018 (reduced by $61.5 \%$ ) and in 2015-17 (reduced by $27.7 \%$ ). Finally, a significant decrease in the NIH-SS score was observed between admission and discharge, in 2018, while NIH-SS values were not available for the reference period 2015-2017.

Clinical and functional rehabilitation outcomes significantly improved for considered measures both in 20152017 and in 2018; however, improvement was significantly higher in 2018 as to TCT improvement, bedsore resolution and bladder catheter removal: a comparison between changes in clinical and functional rehabilitation outcomes in 2015-17 and in 2018 is shown in Table II. 


\section{COPYRIGHT $^{\circledR} 2020$ EDIZIONI MINERVA MEDICA}

$\mathrm{CECCHI}$

IMPLEMENTATION OF A STROKE REHABILITATION ICP

TABLE I.-Patients admitted for stroke rehabilitation in the three-years period 2015-17 and in the first semester of 2018. Clinical and functional characteristics registered at admission and discharge.

\begin{tabular}{|c|c|c|c|c|c|c|c|c|c|}
\hline \multirow[b]{2}{*}{ Parameters } & \multicolumn{4}{|c|}{ Admission } & \multirow{2}{*}{$P$ valuet } & \multicolumn{2}{|c|}{ Discharge } & \multirow{2}{*}{$\begin{array}{l}\text { P value between } \\
\text { admission and } \\
\text { discharge in } \\
2015-2017\end{array}$} & \multirow{2}{*}{$\begin{array}{l}\text { P value between } \\
\text { admission and } \\
\text { discharge in } \\
2018\end{array}$} \\
\hline & N. & $2015-17$ & $\mathrm{~N}$. & 2018 & & $2015-17$ & 2018 & & \\
\hline Age (years) & 443 & $76.6 \pm 11.0$ & 84 & $76.0 \pm 12.9$ & 0.704 & & & & \\
\hline Gender (\% F) & 443 & $47 \%$ & 84 & $54 \%$ & 0.167 & & & & \\
\hline Length of stay (days) & 408 & $36.0 \pm 15.5$ & 84 & $36.6 \pm 17.9$ & 0.800 & & & & \\
\hline Days from the stroke onset & & & 60 & $13.5(28)$ & & & & & \\
\hline RANKIN (score) & 439 & $1.6 \pm 1.6$ & 84 & $1.3 \pm 1.4$ & 0.061 & & & & \\
\hline MMSE (score) & 244 & $23(9)$ & 19 & $25(7)$ & 0.419 & & & & \\
\hline CIRS (score) & 430 & $22.6 \pm 4.3$ & 82 & $22.4 \pm 3.9$ & 0.383 & $22.6 \pm 4.3$ & $22.2 \pm 3.9$ & 0.072 & 0.132 \\
\hline SDC (score) & 425 & $2.7 \pm 1.3$ & 79 & $2.8 \pm 1.3$ & 0.917 & $3.0 \pm 1.2$ & $3.0 \pm 1.3$ & $<0.001$ & 0.001 \\
\hline SAHFE (score) & 358 & $5(1)$ & 79 & $5(1)$ & 0.114 & $4(3)$ & $4(3)$ & $<0.001$ & $<0.001$ \\
\hline Barthel Index (score) & 423 & $30.3 \pm 30.0$ & 82 & $26.4 \pm 26.4$ & 0.286 & $54.0 \pm 32.8$ & $51.5 \pm 31.0$ & $<0.001$ & $<0.001$ \\
\hline TCT (score) & 425 & $48.7 \pm 38.4$ & 81 & $46.2 \pm 37.2$ & 0.567 & $69.6 \pm 33.2$ & $79.0 \pm 31.3$ & $<0.001$ & $<0.001$ \\
\hline NRS (score) & 405 & $1.0 \pm 2.3$ & 76 & $1.1 \pm 2.5$ & 0.298 & $0.4 \pm 1.1$ & $0.4 \pm 1.3$ & $<0.001$ & 0.007 \\
\hline NIHSS (score) & & & 54 & $8.2 \pm 7.2$ & & & $6.3 \pm 7.1$ & & $<0.001$ \\
\hline Bladder catheter $(\% \mathrm{Y})$ & 439 & $34 \%$ & 79 & $52 \%$ & 0.002 & $17 \%$ & $15 \%$ & $<0.001$ & $<0.001$ \\
\hline Bedsore $(\% \mathrm{Y})$ & 441 & $18 \%$ & 82 & $13 \%$ & 0.419 & $13 \%$ & $5 \%$ & 0.001 & 0.003 \\
\hline MMSE (score) & 244 & $23(9)$ & 19 & $25(7)$ & & & & & \\
\hline
\end{tabular}

Data presented as mean $\pm \mathrm{SD}$, median (IQR) or percentage (\%).

SD: Standard deviation; MMSE: mini mental state examination; TOAST: trial of ORG 10172 in acute stroke treatment; CIRS: cumulative illness rating scales; SDC: scale of communicative disability; SAHFE: standardized audit of hip fracture in Europe; TCT: trunk control test; NRS: numerical rating scale; NIHSS: national institutes of health stroke scale.

${ }^{\dagger} \mathrm{P}$ value (two-tailed) between admission in 2015-17 and 2018 based on Bootstrapping with 2000 resamples.

TABLE II.-Comparison between changes in clinical and functional rehabilitation outcomes at discharge in 2015-2017 and in 2018.

\begin{tabular}{|c|c|c|c|}
\hline \multirow{2}{*}{ Changes in clinical and functional rehabilitation } & $2015-17$ & 2018 & \multirow{2}{*}{ P value } \\
\hline & $\begin{array}{c}\text { Mean } \pm \text { SD } \\
\text { N. }(\%)\end{array}$ & $\begin{array}{l}\text { Mean } \pm \text { SD } \\
\text { N. }(\%)\end{array}$ & \\
\hline Improved $\mathrm{mBI}$ between admission and discharge (yes) & $229(54 \%)$ & $52(62 \%)$ & 0.191 \\
\hline TCT at discharge & $69.6 \pm 33.2$ & $79.0 \pm 31.3$ & 0.011 \\
\hline $\mathrm{mBI}$ at discharge & $54.0 \pm 32.8$ & $51.5 \pm 31.0$ & 0.512 \\
\hline Bedsores at discharge (yes) & $58(13 \%)$ & $4(5 \%)$ & 0.033 \\
\hline Bladder catheter at discharge (yes) & $73(17 \%)$ & $13(15 \%)$ & 0.794 \\
\hline Bladder catheters removed during hospitalization & $75(17 \%)$ & $29(37 \%)$ & $<0.001$ \\
\hline
\end{tabular}

Bladder catheters removed during hospitalization

TCT: trunk control test; mBI: modified Barthel Index.

$\lceil\mathrm{P}$ value (two-tailed) based on Bootstrapping with 2000 resamples.

A comparison of discharge destination between 201517 and 2018 is shown in Table III: although we observed a shift from home discharge to resident care discharge in 2018 compared to 2015-2017, no significant difference was found as to discharge destination and, specifically, overall adverse outcomes stayed the same.

\section{Discussion}

This study describes the effect of the implementation of an interdisciplinary developed, evidence based ICP for stroke rehabilitation on postacute stroke inpatient rehabilitation outcomes, in the framework of a continuous quality improvement process. ICPs implementation can be regarded
TABLE III.-Comparison between discharge destination of patients in 2015-2017 and 2018 .

\begin{tabular}{lccc}
\hline Discharge & $2015-17$ & 2018 & P value $^{\dagger}$ \\
\hline Missing values & $82(18.5 \%)$ & $17(20.2 \%)$ & 0.710 \\
Death & $4(0.9 \%)$ & $1(1.2 \%)$ & 0.803 \\
$\begin{array}{l}\text { Discharged to a private } \\
\text { residence }\end{array}$ & $294(66.4 \%)$ & $49(58.3 \%)$ & 0.157 \\
$\begin{array}{l}\text { Discharged to a hospice } \\
\text { Transferred to acute care }\end{array}$ & $2(0.5 \%)$ & $1(1.2 \%)$ & 0.409 \\
$\quad$ hospital & $16(3.6 \%)$ & $4(4.7 \%)$ & 0.613 \\
$\begin{array}{l}\text { Transferred to nursing home } \\
\begin{array}{l}\text { Transferred to another rehab } \\
\quad \text { facility }\end{array}\end{array}$ & $35(7.9 \%)$ & $10(11.9 \%)$ & 0.229 \\
$\begin{array}{l}\text { Voluntary discharge } \\
\text { Total }\end{array}$ & $9(2.0 \%)$ & $1(1.2 \%)$ & 0.187 \\
\hline
\end{tabular}

$\doteqdot \mathrm{P}$ value (two-tailed) based on Bootstrapping with 2000 resamples. 


\section{COPYRIGHT $^{\circledR} 2020$ EDIZIONI MINERVA MEDICA}

as complex interventions, comprising "a number of separate elements which seem essential to the proper functioning of the intervention although the active ingredient of the intervention is difficult to specify." 15 This study focused on the exploratory trial phase, describing the constant and variable components of a replicable intervention and a feasible protocol for comparing the intervention with an appropriate alternative. 15

By this pilot study, we aimed at verifying the acceptance and feasibility of a stroke intensive rehabilitation ICP, as well as its effects on patient-oriented rehabilitation outcomes. Both for ethical and for organizational reasons we chose not to not perform an RCT. This is a limitation of our study, but the impracticalities of applying RCT design to ICPs evaluation studies is well recognized, and beforeand after studies are often regarded "the best available method that may yield useful results" and as such included in dedicated reviews. ${ }^{47,48}$ In designing this study, we complied to the STROBE checklist for observational cohort studies. 49

The ICP was well accepted by the rehabilitation staff, who readily adopted organizational changes and provided constant positive feedback during ICP implementation at weekly team meetings and monthly staff meetings, and overall quite feasible, as the new assessment and procedure were readily incorporated into routine practice, except for an organizational failure to modify the PT records before implementation, that led to incomplete ICP application concerning PT assessment. Staff acceptance and adherence to the ICP was possibly 27,50 related to its previous introduction in 2016 monthly all staff meetings, as a change aimed at improving quality of care; further, the choice of tools and changes of the taking in charge were extensively explained and discussed in both edition of the dedicated formal training course: assessment as a basis for rehabilitation treatment addressed to all health staff and in the 6 dedicated meetings including all MDs, PT coordinators, occupational therapists, speech therapists, and psychologists, all held in the second semester of 2017. As outlined by Williams and Radnor, ${ }^{49}$ a key to acceptance by the health staff was stressing the distinction between standardized care, which is rarely applicable and to the less in rehabilitation, as patients are often complex and care needs might vary considerably, and standardized process employed to deliver healthcare services, such as the proposed ICP, which could help the team to better understand and personalize delivered care within a standardized process frame. Although we did not perform a formal cost evaluation, our findings also suggested the economic sustainability of the ICP, since only a minor additional cost related to MD education on NIH-SS administration was sustained.

As to the comparison of rehabilitation outcomes before and after implementation, similarly to what previously reported in a meta-analysis on effects of stroke management clinical pathways, no difference was found as to serious adverse events (death, re-hospitalization), while, contrary to the same metanalysis, no changes in LOS were observed after the ICP implementation. ${ }^{14}$ The $\mathrm{mBI}$ score increased more after ICP implementation. but the difference was not significant. As to patient satisfaction, that was reported as improved in other studies, ${ }^{14}$ a serious limitation of our study is that we did not directly address patient's satisfaction as one of our desired outcomes: the percentage of voluntary discharges did not vary significantly ( $\mathrm{P}$ value $=0.604)$ between $2018(1.2 \%)$ and 2015-17 (2\%) but this data may be influenced by several confounders hampering its adoption as an indicator of patient's satisfaction. The most interesting result of this pilot study was the definite improvement found after ICP implementation in the resolution of three complexity markers ${ }^{23}, 51$ ): improvement in trunk control, removal of the bladder catheter, and bedsore resolution on discharge. Indeed, the view on ICP in acute stroke management recently performed by Kwan et al., ${ }^{48}$ including six non-randomized studies with 1283 patients, showed that significantly fewer patients suffered urinary tract infections in the care pathway group, possibly confirming a higher effort to address urinary incontinence and risk of urinary infections in the ICP approach. A possible explanation for our results, is that the systematic assessment of these markers of complexity, required to complete the synthesis of the patient problems and IRP objectives in the clinical records, and their systematic check and interdisciplinary discussion at weekly team meetings, may have promoted an awareness and involvement of all team members, rather than primarily PT for trunk control, and nurses, for catheters and bedsores. Team efforts may in turn have produced a prompter, more synergic and more active approach to these patient-centered objectives, ultimately leading to improved outcomes. Indeed, Crook et al. ${ }^{52}$ have shown that perceived health knowledge, information sharing, attitudes, and behavior are related: health information sharing influences behavioral intentions. Thus, the communicative practice of sharing information and objectives promoted by the ICP may reasonably explain the positive impact on specific health outcomes, in agreement to what already found by a review by Allen and Rixon ${ }^{46}$ that ICP in the context of stroke care may "support the timely imple- 


\section{COPYRIGHT $^{(\odot)} 2020$ EDIZIONI MINERVA MEDICA}

mentation of clinical interventions and the mobilization of resources around the patient without incurring additional increases in length of stay." Examples of synergic interventions that were observed to be better scheduled and coordinated after the ICP implementation include prompter prescription of nutritional support and incremented mobilization for bedsores; scheduling of urological protocol for bladder catheter removal right on admission, when possible, and prompter prescription of medication to promote bladder function, in the attempt to allow urinary catheter removal. As to TCT improvement, a better control of patients positioning by teaming of healthcare assistants, PT and occupational therapists, and prompter shift to less assistive chair as soon as trunk control was recovered was observed. TCT is known to be a strong early predictor of activities of daily living recovery, thus, early assessment and management of trunk control after stroke is highly recommended. ${ }^{33}$ In our study, we found that $\mathrm{mBI}$ changes were also higher after ICP implementation, but this difference was not significant; possibly, a longer time frame of observation by a 6 months follow up (that we have now scheduled) my allow us to detect whether the ICP-associated TCT improvement at discharge may eventually be associated to better long term functional recovery.

Although these results are very encouraging, the limitations of evaluating a complex intervention by a pilot study must be taken into account: indeed, as reported by Kwan et al. ${ }^{48}$ effects may be smaller or more variable and response rates may be lower when the ICP is implemented across a wider range of settings. Further, the investigators who assessed the outcomes were not blinded to the ICP implementation, we failed to collect a direct, reliable measure of patients' satisfaction, and no follow-up was performed. Further, these before-and-after analyses might also be hindered by imbalance of prognostic factors and case mix at baseline, but, to ensure that the unequal sample sizes between the two time periods would not affect statistical significance, we carried out a further statistical analysis (bias-corrected bootstrapping procedure). Besides, our populations, although different in size, were remarkably similar in terms of clinical and functional complexity on admission, except for a higher percentage of women admitted in 2018 vs. 2015-17; however, since women seem to be at equal or even heightened risk for poor outcomes after stroke rehabilitation, ${ }^{54}$ this difference would hardly produce a positive bias as to the effects of ICP implementation. Other limitations of this pilot study include a relatively short time period of evaluation after implementation and local experience, that limits generalizability of results, even though, to minimize the last issue, two different rehabilitation facilities were involved.

\section{Limitations of the study}

This study also revealed some of the ICP weaknesses and implementation failures, that shall be specifically addressed in the next step of ICP implementation on a larger scale. To promote further implementation, the structured assessment tools must be included in the next reprint of PT records. This shall address failure of implementation of the PT assessment. More detailed recording of clinical outcomes, including for instance dysphagia, shall be provided, and a formal follow up assessment to evaluate long term functional and clinical outcomes shall be scheduled. As to formal patient involvement, the ICP shall be proposed to and revised with representatives of the stroke patients Italian organization, and both a 6-months follow up and a systematic assessment of patients' satisfaction on discharge shall be introduced in further ICP implementation.

\section{Conclusions}

An evidence based ICP for Stroke rehabilitation was interdisciplinary developed by a multiregional Italian Health group. The ICP provided a standard assessment and standardized processes of care delivery to stroke patients in the involved rehabilitation centers. Its implementation in two pilot facilities supported its acceptability and feasibility. Compared to prior practice, ICP implementation was associated to a significant improvement in some patientoriented outcomes at discharge: recovery of trunk control, bladder catheter removal, and pressure sore resolution. This knowledge-translation experience in rehabilitation, involving a multidisciplinary group of health professionals, will allow multicenter studies and quality benchmarking, as the stroke rehabilitation ICP will be implemented on a larger scale.

\section{References}

1. van Wijck F, Bernhardt J, Billinger SA, Bird ML, Eng J, English C, et al. Improving life after stroke needs global efforts to implement evidencebased physical activity pathways. Int J Stroke 2019;14:457-9.

2. Skolarus LE, Burke JF, Brown DL, Freedman VA. Understanding stroke survivorship: expanding the concept of poststroke disability. Stroke 2014;45:224-30.

3. Gadidi V, Katz-Leurer M, Carmeli E, Bornstein NM. Long-term outcome poststroke: predictors of activity limitation and participation restriction. Arch Phys Med Rehabil 2011;92:1802-8. 


\title{
COPYRIGHT $^{(\odot)} 2020$ EDIZIONI MINERVA MEDICA
}

\author{
IMPLEMENTATION OF A STROKE REHABILITATION ICP
}

CECCHI

4. Winstein CJ, Stein J, Arena R, Bates B, Cherney LR, Cramer SC, et al.; American Heart Association Stroke Council, Council on Cardiovascular and Stroke Nursing, Council on Clinical Cardiology, and Council on Quality of Care and Outcomes Research. Guidelines for Adult Stroke Rehabilitation and Recovery: A Guideline for Healthcare Professionals From the American Heart Association/American Stroke Association. Stroke 2016;47:e98-169.

5. Lindsay P, Furie KL, Davis SM, Donnan GA, Norrving B. World Stroke Organization global stroke services guidelines and action plan. Int J Stroke 2014;9(Suppl A100):4-13.

6. Shortell SM, O’Brien JL, Carman JM, Foster RW, Hughes EF, Boerstler $\mathrm{H}$, et al. Assessing the impact of continuous quality improvement/ total quality management: concept versus implementation. Health Serv Res 1995;30:377-401.

7. Connell LA, Smith MC, Byblow WD, Stinear CM. Implementing biomarkers to predict motor recovery after stroke. NeuroRehabilitation 2018;43:41-50.

8. Kwakkel G, Lannin NA, Borschmann K, English C, Ali M, Churilov L, et al. Standardized measurement of sensorimotor recovery in stroke trials: Consensus-based core recommendations from the Stroke Recovery and Rehabilitation Roundtable. Int J Stroke 2017;12:451-61.

9. WHO Consultative Group on Equity and Universal Health Coverage. Making fair choices on the path to universal health coverage: Final report of the WHO Consultative Group on Equity and Universal Health Coverage. Health Econ Policy Law 2014;1-7.

10. Piano di indirizzo per la Riabilitazione. Gazzetta Ufficiale; 2011 [Internet]. Available form: https://www.gazzettaufficiale.it/eli/ $\mathrm{id} / 2011 / 03 / 02 / 11 \mathrm{~A} 02720 / \mathrm{sg}$ [cited 2019, Oct 5].

11. La centralità della Persona in riabilitazione: nuovi modelli organizzativi e gestionali. Quad del Minist della Salut 2011;8:74-9.

12. Lenti G, Agosti M, Massucci M, Zampolini M, Paolucci S, Franceschini M. Developing a minimum data set for stroke patients assessment: the "Protocollo di Minima per l'Ictus (PMIC) as a starting point towards an Italian stroke registry. Eur J Phys Rehabil Med 2008;44:263-9.

13. Brennan S, Mckenzie JE, Whitty P, Buchan H, Green S, Bosch M, et al. Continuous quality improvement: effects on professional practice and healthcare outcomes in primary care. Cochrane Database Syst Rev 2017;2017.

14. Huang D, Song X, Tian J, Cui Q, Yang K. Effects of clinical pathways in stroke management: A meta-analysis. Neurol Asia 2015;20:335-42.

15. Campbell H, Hotchkiss R, Bradshaw N, Porteous M. Integrated care pathways. BMJ 1998;316:133-7.

16. Currie VL, Harvey G. The use of care pathways as tools to support the implementation of evidence-based practice. J Interprof Care 2000;14:311-23.

17. Wensing M, Laurant M, Ouwens M, Wollersheim H. Organizational implementation strategies for change. In: Improving Patient Care. Hoboken, NJ: Wiley; 2013. p.240-53.

18. Everink IH, van Haastregt JC, Maessen JM, Schols JM, Kempen GI. Process evaluation of an integrated care pathway in geriatric rehabilitation for people with complex health problems. BMC Health Serv Res 2017; 17:34

19. The International Classification of Functioning, Disability and Health (ICF). World Health Organization; 2001 [Internet]. Available from: https://www.cdc.gov/nchs/data/icd/icfoverview_finalforwho10sept.pdf [cited 2019, Oct 27].

20. National Guidelines Clearinghouse. AHCPR [Internet]. Available from: https://www.ahrq.gov/gam/index.html [cited 2019, Oct 27].

21. Guidelines International Network. GIMBE; 2020 [Internet]. Available from: https://g-i-n.net/ [cited 2019, Oct 27].

22. The AGREE II Instrument; AGREE Next Steps Consortium. 2017 [Internet]. Available from: www.agreetrust.org/wp-content/uploads/2017/12/ AGREE-II-Users-Manual-and-23-item-Instrument-2009-Update-2017. pdf [cited 2019, Apr 1].
23. Bernardini B. Iper2: Indicatori di processo e di esito in riabilitazione. Iper2; [Internet]. Available from: www.iper2.it [cited 2019, Oct 27].

24. Heart and Stroke Foundation. Canadian Partnership for Stroke recovery. Stroke Engine; [Internet]. Available from: https://www.strokengine. ca/en/ [cited 2019, Oct 27].

25. Basaglia N. Medicina riabilitativa: medicina fisica e riabilitazione: principi e pratica. Naples: Idelson-Gnocchi; 2009.

26. Cartabellotta A. Position Statement GIMBE ${ }^{\circledR}$ : Il Governo Clinico nelle Aziende Sanitarie. GIMBE; [Internet]. Available from: www.gimbe. org/gimbe/statement/ps_gc.htm [cited 2019, Oct 27].

27. Moore L, Hallingberg B, Wight D, Turley R, Segrott J, Craig P, et al. Exploratory studies to inform full-scale evaluations of complex public health interventions: the need for guidance. J Epidemiol Community Health 2018;72:865-6.

28. Vanhaecht K, De Witte K, Depreitere R, Sermeus W. Clinical pathway audit tools: a systematic review. J Nurs Manag 2006;14:529-37.

29. Linn BS, Linn MW, Gurel L. Cumulative illness rating scale. J Am Geriatr Soc 1968;16:622-6.

30. Folstein MF, Folstein SE, McHugh PR. "Mini-mental state". A practical method for grading the cognitive state of patients for the clinician. J Psychiatr Res 1975;12:189-98.

31. Collin C, Wade D. Assessing motor impairment after stroke: a pilot reliability study. J Neurol Neurosurg Psychiatry 1990;53:576-9.

32. Parker MJ, Currie CT, Mountain JA, Thorngren KG. Standardised audit of hip fracture in Europe (SAHFE). Hip Int 1998;8:10-5.

33. Meinecke C, Cappadonia C, Bernardini B. Validazione di una semplice scala di impairment comunicativo nell'anziano. Atti del XXIII Congresso Nazionale SIMFER Valutazione e Qualità dell'Assistenza in Medicina Riabilitativa. Ancona: Abstract Book; 1995. p.56-7.

34. Krasner D. AHCPR Clinical Practice Guideline Number 15, Treatment of Pressure Ulcers: a pragmatist's critique for wound care providers. Ostomy Wound Manage 1995;41(Suppl):97S-101S, discussion 102S.

35. Bergstrom N, Braden BJ, Laguzza A, Holman V. The braden scale for predicting pressure sore risk. Nurs Res 1987;36:205-10.

36. Dworkin RH, Turk DC, Farrar JT, Haythornthwaite JA, Jensen MP, Katz NP, et al.; IMMPACT. Core outcome measures for chronic pain clinical trials: IMMPACT recommendations. Pain 2005;113:9-19.

37. Shah S, Vanclay F, Cooper B. Improving the sensitivity of the Barthel Index for stroke rehabilitation. J Clin Epidemiol 1989;42:703-9.

38. Galeoto G, Mollica R, Astorino O. Italian translation, adaptation and validation. Int J Neurol Neurother 2015;2:1-7.

39. Demeurisse G, Demol O, Robaye E. Motor evaluation in vascular hemiplegia. Eur Neurol 1980;19:382-9.

40. van Swieten JC, Koudstaal PJ, Visser MC, Schouten HJ, van Gijn J. Interobserver agreement for the assessment of handicap in stroke patients. Stroke 1988;19:604-7.

41. Brott $\mathrm{T}$, Adams HP Jr, Olinger CP, Marler JR, Barsan WG, Biller J, et al. Measurements of acute cerebral infarction: a clinical examination scale. Stroke 1989;20:864-70.

42. Fiorella M, Pezzella F, Lyden P, Emr M, Warren M. Training and online certification on the NIH-SS - Italian. Health of Care Point; [Internet] Available from: https://secure.trainingcampus.net/UAS/Modules/TREES/ windex.aspx [cited 2020, Jun 9].

43. Bamford J, Sandercock P, Dennis M, Burn J, Warlow C. Classification and natural history of clinically identifiable subtypes of cerebral infarction. Lancet 1991;337:1521-6.

44. Guralnik JM, Simonsick EM, Ferrucci L, Glynn RJ, Berkman LF, Blazer DG, et al. A short physical performance battery assessing lower extremity function: association with self-reported disability and prediction of mortality and nursing home admission. J Gerontol 1994;49:M85-94.

45. Hoffmann TC, Glasziou PP, Boutron I, Milne R, Perera R, Moher D, et al. Better reporting of interventions: template for intervention description and replication (TIDieR) checklist and guide. BMJ 2014;348. 


\section{COPYRIGHT $^{(\odot)} 2020$ EDIZIONI MINERVA MEDICA}

CECCHI

IMPLEMENTATION OF A STROKE REHABILITATION ICP

46. Dwivedi AK, Mallawaarachchi I, Alvarado LA. Analysis of small sample size studies using nonparametric bootstrap test with pooled resampling method. Stat Med 2017;36:2187-205.

47. Allen D, Rixson L. How has the impact of "care pathway technologies' on service integration in stroke care been measured and what is the strength of the evidence to support their effectiveness in this respect? Int J Evid-Based Healthc 2008;6:78-110.

48. Kwan J, Sandercock P. In-hospital care pathways for stroke: a Cochrane systematic review. Stroke 2003;34:587-8.

49. von Elm E, Altman DG, Egger M, Pocock SJ, Gøtzsche PC, Vandenbroucke JP; STROBE Initiative. The Strengthening the Reporting of Observational Studies in Epidemiology (STROBE) statement: guidelines for reporting observational studies. J Clin Epidemiol 2008;61:344-9.
50. Williams S, Radnor Z. An integrative approach to improving patient care pathways. Int J Health Care Qual Assur 2018;31:810-21.

51. Cecchi F, Pancani S, Antonioli D, Avila L, Barilli M, Gambini M, et al. Predictors of recovering ambulation after hip fracture inpatient rehabilitation. BMC Geriatr 2018;18:201.

52. Crook B, Stephens KK, Pastorek AE, Mackert M, Donovan EE. Sharing Health Information and Influencing Behavioral Intentions: The Role of Health Literacy, Information Overload, and the Internet in the Diffusion of Healthy Heart Information. Health Commun 2016;31:60-71.

53. Hsieh CL, Sheu CF, Hsueh IP, Wang CH. Trunk control as an early predictor of comprehensive activities of daily living function in stroke patients. Stroke 2002;33:2626-30.

54. Appelros P, Stegmayr B, Terént A. A review on sex differences in stroke treatment and outcome. Acta Neurol Scand 2010;121:359-69.

Conflicts of interest.-The authors certify that there is no conflict of interest with any financial organization regarding the material discussed in the manuscript. Funding.-This study was supported by the Italian Ministry of health (current research funds).

Authors' contributions.-All authors read and approved the final version of the manuscript.

History.-Manuscript accepted: September 14, 2020. - Manuscript revised: September 2, 2020. - Manuscript received: January $27,2020$.

Supplementary data.-For supplementary materials, please see the HTML version of this article at www.minervamedica.it 